\title{
Post Hysterectomy Fallopian Tube Prolapse: A Rare Case
}

Dr. Jadhav Vaishali ${ }^{1 *}$, Dr Mishra Nigamanand ${ }^{2}$, Dr. Savani Gayatri ${ }^{3}$, Dr. G Geetadevi ${ }^{4}$, Dr. Gaddam Prachi ${ }^{5}$

\footnotetext{
${ }^{1-4}$ Dept of Gynaec/Obst, ${ }^{5}$ Dept of Pathology, Obstetrics \& Gynecology Department BARC Hospital Anushakti Nagar Mumbai- 400094 India

*Corresponding author: Dr. Jadhav Vaishali

Abstract

Fallopian tube prolapse through the vaginal vault is a complication seen in few cases of hysterectomies where, the adnexa is preserved. The overall occurrence of fallopian tube prolapse through vaginal vault after hysterectomy is $0.01-0.05 \%$ regardless of the route [1].The only means of definitive diagnosis is the histopathological examination. Fallopian tube prolapse can be prevented by prophylactic salpingectomy or by suturing the adnexa high up in the pelvis in abdominal hysterectomy. This condition can be confused with vault granulation tissue and serious condition like carcinoma in vaginal vault. Here, we present a case of $60 y$ rs old lady with fimbrial prolapse through the vault.

Keywords: Fallopian tube prolapse, Post-hysterectomy, Vaginal vault, Granulation tissue.

Copyright $\odot 2020$ The Author(s): This is an open-access article distributed under the terms of the Creative Commons Attribution 4.0 International License (CC BY-NC 4.0) which permits unrestricted use, distribution, and reproduction in any medium for non-commercial use provided the original author and source are credited.
}

\section{INTRODUCTION}

Fallopian tube prolapse is more commonly observed following vaginal hysterectomy compared to abdominal hysterectomy. Pozzi in 1902 first reported 2 cases of fallopian tube prolapse after vaginal hysterectomy [2]. The first two cases to occur after abdominal hysterectomy was described by Funnel et al. in 1955 [3]. The condition is a very rare complication with about 100 cases in literature being reported. Patient can present immediately after the hysterectomy or even as late as $32 \mathrm{yrs}$.It is often misdiagnosed as granulation tissue leading to delayed treatment. Fallopian tube prolapse should be considered in all cases of pelvic or abdominal pain accompanied by vaginal bleeding or discharge after hysterectomy, with or without granulation tissue in the vaginal vault. This can present after abdominal, laparoscopic and vaginal hysterectomy. Aetiology can be multifactorial including postoperative fever, poor condition of patient, vault hematoma and non-closure, early resumption of sexual activity, insufficient vaginal preparation preoperatively, difficult surgical procedure, profuse postoperative vaginal discharge, use of intraoperative vaginal drains and packs, chronic cough, diabetes mellitus, constipation, steroids, or even malignancy.

\section{Case Report}

A 60years old female Para2 Living2 came to the outpatient department with complaints of pain in abdomen and white discharge since $2-3$ months. She is a known case of hypertension and diabetes on medications.

Patient has $\mathrm{H} / \mathrm{O}$ abdominal hysterectomy done 20 years back in view of menorrhagia. Post-operatively she had serous discharge from the abdominal wound which was managed conservatively and healed completely. Now on per speculum examination there was presence of a reddish pink fleshy mass at the vault with minimum discharge but no bleeding. Vault smear was taken and dysplasia ruled out.

Pelvic ultrasound showed left adnexal simple cyst mostly ovarian $(3.5 \times 2.8 \mathrm{~cm})$.

Relevant tumour markers were done and were normal.

Patient was posted for laparoscopic bilateral salpingo-oophorectomy with excision of the probable vault granulation tissue.

\section{Intraoperative findings}

Bilateral tubes and ovaries were found embedded between dense adhesions of bowel loops. Left ovarian cyst 3-4 cm, containing serous fluid. Bilateral oophorectomy was done with right salpingectomy. Left tube could not be identified due to adhesions. The fleshy mass was excised vaginally and sent for histopathological examination after labelling.

\section{Histopathological report}

Section from vaginal vault showed bits of fallopian tube epithelium surrounded by fibrosis and chronic inflammation. 


\section{Microscopic picture of the slide}

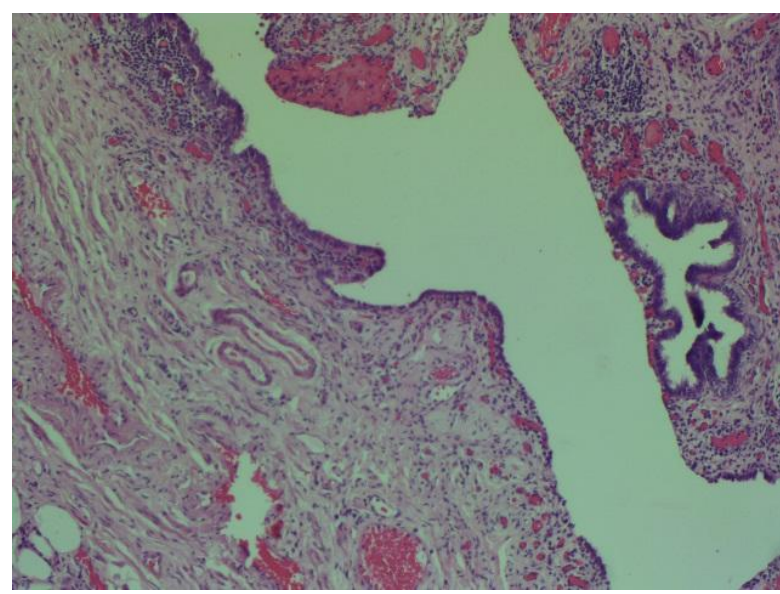

Fig-1: Micrograph shows tubal epithelium in plicae and glands with adjacent chronic inflammation (H\&EX100)

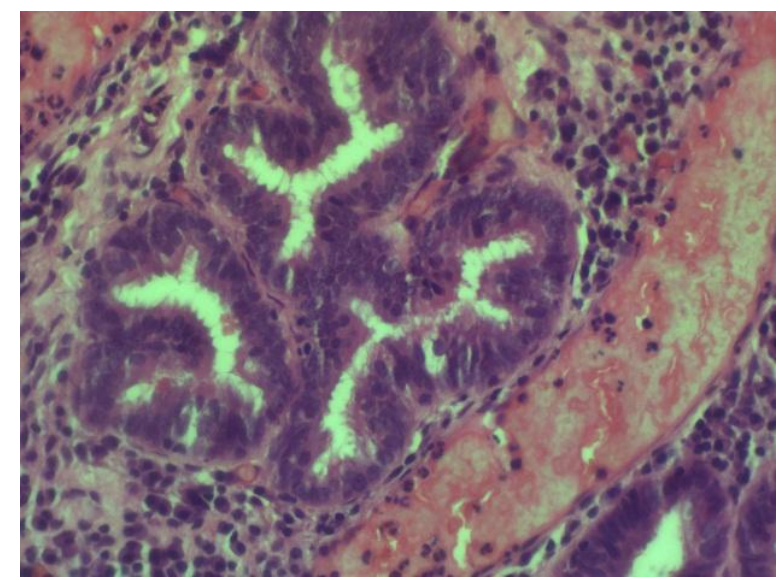

Fig-2: Micrograph shows tubal lining by pesudostratified ciliated columnar epithelium (H\&EX400)

\section{DISCUSSION}

The incidence of fimbrial tube prolapse is $0.5 \%$ with vaginal hysterectomy and $0.06 \%$ with abdominal hysterectomy and $0 \%$ with laparoscopic hysterectomies. The fallopian tube prolapse being a very rare complication post hysterectomy is reported to around 100 cases. In literature about $2 / 3$ rd of cases have occurred after abdominal hysterectomy and more in premenopausal women. The prerequisite for the condition to occur is the communication between peritoneal cavity and vagina, the sufficient length and mobility of the fallopian tube, defective operative technique, and difficulty in closing the vaginal vault. In addition, the left fallopian tube may move close to a small opening in the vaginal cuff, and the pressure gradient between the abdominal cavity and vagina may facilitate the tubal prolapse. Confirmed diagnosis is on histopathology. When the fallopian tube epithelium is not visualized it is the immunohistochemistry (IHC) that helps. Pan keratin is an immunostain that marks broad array of keratins.IHC for pan keratin is used [3]. The mean time interval between hysterectomy and development of fimbrial prolapse is about 4 months, and the longest interval is 32 years [4]. Patients usually present with profuse vaginal discharge either watery, purulent, foul smelling, or bloody discharge associated with pain abdomen and dyspareunia. Often it is misdiagnosed most commonly as granulation tissue [5]. Other common diagnosis is vaginal adenosis, endometriosis, and primary metastatic adenocarcinoma. A diagnosis of vaginal vault granulation tissue should be concluded if the tissue is found to be resistant to cauterization. Hysterectomies in which the adnexa is preserved, histopathological examination helps in the definitive diagnosis. Fimbrial structures can be recognised either grossly or microscopically due to evidence of columnar epithelium along with smooth muscle fibers [6]. Vesicovaginal fistula is ruled out by cystoscopy. There is no ideal surgical method that is described. Excisional biopsy is the only means of diagnosis and treatment. Total salpingectomy with closure of vault defect is considered the optimal management. Many a time's partial salpingectomy is performed due to dense adhesions. Patient maybe symptomatic due to traction on remaining part of the tube, hence total salpingectomy is preferred [7]. Poor healing due to various factors like post op vault hematoma, fever, infection, non- closure of vault and early resumption of sexual activity increases the chances of fallopian tube prolapse [8]. Prophylactic salpingectomies would prevent tubal and serous ovarian malignancy [9]. Differential diagnosis of such microscopic findings includes cysts of mesonephric or paramesonephric duct, vaginal adenosis, endometriosis, primary and metastatic adenocarcinoma [10].

\section{Conclusion}

Any conservative hysterectomies should be accompanied with prophylactic salpingectomies to prevent fallopian tube prolapse and also prevent tubal and serous ovarian carcinoma. Not to fix cornual stump for vault suspension. Other predisposing factors such as malnutrition, poorly controlled diabetes mellitus, chronic cough and chronic constipation should be taken care of. To avoid resumption of sexual intercourse before complete healing of vaginal cuff should be suggested. Fallopian tube prolapse is one of the differential diagnoses. Post-operative vault infection, haematoma, malignancy and tissue radiation are other causes of vaginal bleeding, discharge or pain which should be ruled out.

\section{REFERENCES}

1. Fan QB, Liu ZF, Lang JH, Sun DW, Leng JH, Zhu L. Fallopian tube prolapsed following hysterectomy. Chin Med Sci J. 2006; 2:20-3.

2. Pozzi M. Hernia of the tube into the vagina at a scar operative vaginal hysterectomy. CR Soc Obstet Gynecol Paediatr Paris. 1902; 4:255.

3. Zutshi V, Aggarwal P, Batra S. Post-hysterectomy fallopian tube prolapsed: Elementary yet enigmatic. J Turkish-German Gnaecol Assoc. 2008; 117-9. 
4. Ouldamer L, Caille A, Body G. Fallopian tube prolapse after hysterectomy: Asystematic review. PLoS One 2013; 8:e76543.

5. Pankeratin AB, Sung YS, Kang JS, Park MH. Fallopian tube prolapsed misdiagnosed as vault granulation tissue: a report of three cases. Pathol Res Pract.2005; 201(12):819-2.

6. Sanklecha VM, Sisodia SM, Ansari SA, Pol S. Posthysterectomy Fallopian tube prolapse. J Midlife Health. 2012; 3:40-1.

7. Caceres A, McCarus SD. Fallopian tube prolapse after Laparoscopic hysterectomy. Obstet Gynecol.2008; 112(2):494-5.
8. Quezada Y, Karram M, Whiteside JL. Case report: Diagnosis and management of peritoneovaginal fistula. J Minim Invasive Gynecol. 2015; 22:134.

9. Alarab M, Foley M, Fennelly D. Prolonged survival in two cases of carcinoma of the fallopian tube presenting after hysterectomy: Case reports. Eur Oncol. 2003;24:91-2

10. Jashnani KD, Naik LP. Prolapsed fallopian tube with squamous metaplasia. J Postgrad Med. 2002;48:241-2. 